\title{
CARACTERÍSTICAS ESTRUTURAIS E PRODUTIVAS EM HÍBRIDOS INTRAESPECÍFICOS E INTERESPECÍFICOS DE CAPIM-ELEFANTE
}

\section{STRUCTURAL AND PRODUCTIVE CHARACTERISTICS IN INTRASPECIFIC AND INTERSPECIFIC HYBRIDS OF ELEPHANTGRASS}

\author{
João Virgínio Emerenciano Neto ${ }^{1 *}$ ORCID - http://orcid.org/0000-0003-3060-9696 \\ Marcio Gleybson da Silva Bezerra² ORCID - https://orcid.org/0000-0001-9866-6617 \\ Alan Ferreira de França ${ }^{3}$ ORCID - https://orcid.org/0000-0001-8195-4997 \\ Emerson Moreira de Aguiar 4 ORCID - http://orcid.org/0000-0002-7088-5479 \\ Gelson dos Santos Difante ${ }^{5}$ ORCID - https://orcid.org/0000-0001-6610-8952 \\ ${ }^{1}$ Universidade Federal do Vale do São Francisco, Petrolina, PE, Brasil. \\ ${ }^{2}$ Universidade Federal Rural do Semi-Árido, Mossoró, RN, Brasil. \\ 3Universidade Federal Rural de Pernambuco, Garanhuns, PE, Brasil. \\ ${ }^{4}$ Universidade Federal do Rio Grande do Norte, Macaíba, RN, Brasil. \\ ${ }^{5}$ Universidade Federal do Mato Grosso do Sul, Campo Grande, MS, Brasil. \\ *Autor para correspondência - joao neto@zootecnista.com.br
}

\section{Resumo}

Objetivou-se avaliar a estrutura e a produção de forragem em híbridos de capim-elefante manejados sob corte. Utilizaram-se como tratamentos 24 clones de capim-elefante provenientes da Embrapa Gado de Leite e o Cameroom como testemunha. Os capins foram cortados rentes ao solo, a cada 60 dias. Os parâmetros avaliados foram: produção e teor de matéria seca, número de perfilhos basais, número de folhas por perfilho, altura da planta, diâmetro do colmo. A maior altura das plantas foi observada no clone CNPGL 00-103-1. O grupo com maior número de perfilhos teve três clones e média de 39,38 perfilhos $\mathrm{m}^{-2}$. No grupo dos clones CNPGL 00-103-1, CNPGL 93-25-3, CNPGL 0016-1 e CNPGL 00-90-3 foram observados os maiores teores de matéria seca (22,7\% de MS). As maiores massas de forragem, de lâmina foliar e de colmo foram observadas no clone CNPGL 00-214, 15852 e $6195 \mathrm{~kg} \mathrm{ha}^{-1}$ de MS respectivamente. A maior relação lâmina foliar/colmo foi do clone CNPGL 00-201-1 e apenas neste a massa de lâmina foliar foi superior à de colmo. O clone CNPGL 00-214 mostrou-se mais produtivo, com alta capacidade de perfilhamento e de acúmulo de forragem. Palavras-chave: Colmo; lâmina foliar; perfilho; Pennisetum sp.

\begin{abstract}
The objective of this study was to evaluate the structure and forage production of hybrids of elephantgrass managed under cut. Twenty-four clones of elephant-grass from Embrapa dairy cattle were used as treatments, and Cameroom as a control. The grasses were cut close to the ground, every 60 days. The parameters evaluated were yield and dry matter content, number of basal tillers, number of leaves per tiller, plant height, stem diameter. The highest height of the plants was observed in clone CNPGL 00-103-1. The group with the highest number of tillers had three clones and a mean of $39.38{\text { tiller } \mathrm{m}^{-}}^{-}$
\end{abstract}


2. In the group of clones CNPGL 00-103-1, CNPGL 93-25-3, CNPGL 00-16-1 and CNPGL 00-90-3 the highest dry matter contents $(22.7 \% \mathrm{DM})$ were observed. The highest masses of forage, leaf blade and stem were observed in clone CNPGL 00-214, 15852 and $6195 \mathrm{~kg} \mathrm{ha}^{-1} \mathrm{DM}$, respectively. The highest leaf blade / stem ratio was of the CNPGL 00-201-1 clone, and only in this did the leaf blade mass exceed that of the stem. Clone CNPGL 00-214 showed to be more productive, with high tillering capacity and forage accumulation.

Keywords: Leaf blade; Pennisetum sp; stem; tiller.

Recebido em: 07 de maio de 2017.

Aceito em: 07 de junho de 2019

\section{Introdução}

O capim-elefante (Pennisetum purpureum Schum.) é uma planta forrageira perene com elevada produtividade, encontra-se distribuído pelos diferentes ecossistemas brasileiros, utilizado sob corte e pastejo $^{(1)}$. Programas de melhoramento de espécies forrageiras têm sido utilizados para aumentar a produção de forragem. A hibridação é um mecanismo bastante utilizado nesses programas, pode ser feita envolvendo cultivares ou variedades de uma mesma espécie (intraespecífica) ou envolvendo diferentes espécies (interespecífica), como a do capim-elefante com o milheto (Pennisetum glaucum), bastante utilizada para este propósito ${ }^{(2)}$. Esses programas visam à obtenção de cultivares que apresentem características desejáveis tanto para capineiras como pastejo, propagação por sementes, adaptação a solos de baixa fertilidade, redução da sazonalidade da produção, melhor composição química da forragem, maior teor de matéria seca (favorece a ensilagem), além de maior produtividade em relação às variedades já cultivadas ${ }^{(3)}$.

Os vários estudos com capim-elefante revelam que tanto a escolha da cultivar a ser utilizada como sua adaptação às condições edafoclimáticas e ao manejo empregado são indispensáveis ao seu desempenho $^{(4)}$. Tendo em vista que a produção é muito heterogênea entre híbridos, Silva et al. ${ }^{(5)}$ estimaram uma produtividade em clones de capim-elefante que varia de 2,1 a 14,0 tha ${ }^{-1}$ de MS a cada 60 dias, que pode ainda ter comportamento distinto dos genótipos nos diferentes ambientes. Diante disso, objetivou-se identificar características estruturais e produtivas em clones de capim-elefante intra e interespecíficos.

\section{Materiais e métodos}

O experimento foi realizado na Estação Experimental Rommel Mesquita de Farias, município de Parnamirim/RN, pertencente à Empresa de Pesquisa Agropecuária do Rio Grande do Norte EMPARN. A Estação apresenta como coordenadas geográficas: latitude 554'56" S e longitude $35^{\circ} 15^{\prime} 46$ " O e altitude de 10 metros acima do nível do mar.

O clima da região, de acordo com a classificação climática de Thornthwaite ${ }^{(7)}$, é subúmido seco, com excedente hídrico de maio a agosto. A precipitação média anual é de $1048 \mathrm{~mm}$ e evapotranspiração potencial média acumulada anual de $1472 \mathrm{~mm}$. A precipitação e a temperatura ambiental na área 
foram monitoradas durante o período experimental (Figura 1).

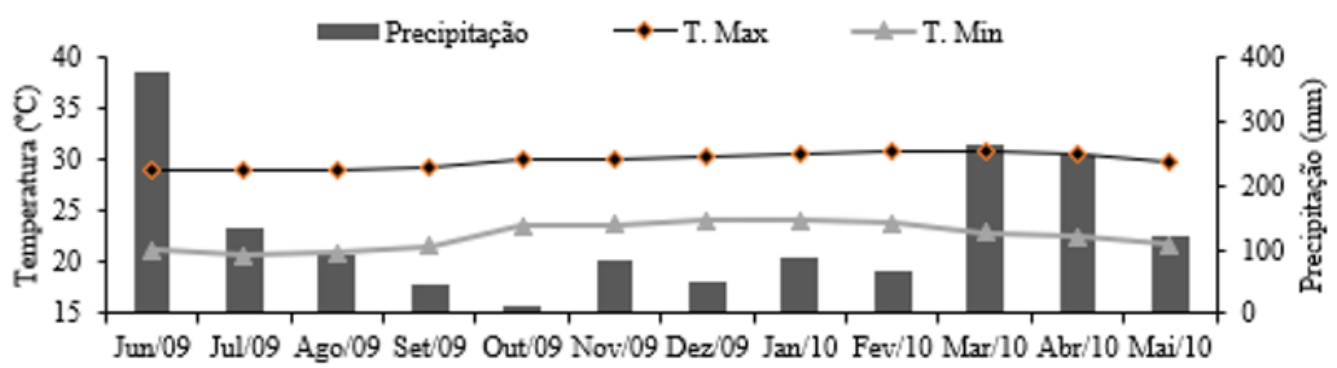

Figura 1- Precipitação pluviométrica e temperaturas: máxima (T. Máx.) e mínima (T. Mín.) do ar na área experimental entre junho de 2009 e maio de 2010

O solo da área experimental foi classificado como Neossolo Quartzarênico ${ }^{(6)}$ de baixa fertilidade,

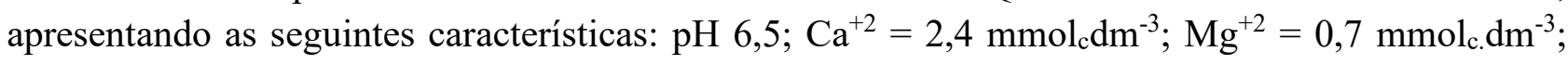
$\mathrm{Al}^{+3}=0,0 \mathrm{mmol}_{\mathrm{c}} \cdot \mathrm{dm}^{-3} ; \mathrm{P}=3,0 \mathrm{mmol}_{\mathrm{c}} \cdot \mathrm{dm}^{-3} ; \mathrm{K}^{+}=8,0 \mathrm{mmol}_{\mathrm{c}} \mathrm{dm}^{-3}$. Após o corte de uniformização e após o segundo corte foram realizadas adubação com $20 \mathrm{t} \mathrm{ha}^{-1}$ de esterco bovino e com $50 \mathrm{~kg} \mathrm{ha}^{-1} \mathrm{de}$ $\mathrm{K}_{2} \mathrm{O}$ (cloreto de potássio) e $50 \mathrm{~kg} \mathrm{ha}^{-1}$ de $\mathrm{N}$ (sulfato de amônio), além de uma adubação fosfatada anual de $100 \mathrm{~kg} \mathrm{ha}^{-1}$ de $\mathrm{P}_{2} \mathrm{O}_{5}$ (superfosfato simples).

Os clones foram plantados em março de 2009 e o corte de uniformização realizado 90 dias após o plantio, para avaliação foram seis cortes (a cada 60 dias), finalizando em maio de 2010. A área experimental foi montada em 12 linhas de 25,0 m espaçadas por 1,5 m entre si, sendo duas linhas para bordadura e 10 para avaliação, totalizando 50 parcelas (duas por tratamento). Cada parcela tinha 5,0 $\mathrm{m}$ de comprimento por 1,5 $\mathrm{m}$ de largura (7,5 $\mathrm{m}^{2}$ de área útil). Nos meses de setembro, outubro, dezembro e janeiro, foi realizada complementação hídrica por aspersão, para garantir uma quantidade mensal de água mínima de $80 \mathrm{~mm}$. Não ocorreram ataques de pragas ou doenças durante a condução do ensaio.

Os tratamentos avaliados foram 24 clones de capim-elefante provenientes da Embrapa Gado de Leite e o Cameroon como testemunha (Tabela 1).

A massa de forragem (MFO, $\mathrm{kg} \mathrm{ha}^{-1}$ de MS) foi obtida pela forragem contida na área útil da parcela, cortada manualmente rente ao solo e posteriormente pesada em balança digital. Do material colhido, retirou-se uma amostra em torno de $500 \mathrm{~g}$ (perfilhos inteiros), cortados em pedaços de 3 a $4 \mathrm{~cm}$, pesados e colocados em estufa com ventilação forçada a $55^{\circ} \mathrm{C}$ até peso constante para determinação da matéria seca.

Para avaliação da massa dos componentes morfológicos da forragem e a relação lâmina foliar/colmo foram retiradas subamostras representativas das amostras colhidas para a determinação da massa de forragem, que posteriormente foram separadas manualmente nas frações de lâmina foliar e colmo (colmo + bainha). Após a separação, os componentes foram pesados e secos em estufa de circulação forçada de ar a $55^{\circ} \mathrm{C}$ até peso constante para determinação da matéria seca. 
Tabela 1- Classificação genética e porte dos clones avaliados

\begin{tabular}{|c|c|c|c|}
\hline Clone & Tipo de Clone & Cromossomos & Porte \\
\hline CNPGL 00-103-1 & Híbrido Interespecífico & Triplóide $^{1}$ & Normal \\
\hline CNPGL 00-108-1 & Híbrido Interespecífico & Triplóide $^{1}$ & Normal \\
\hline CNPGL 00-1-1 & Híbrido Intraespecífico & Tetraplóide & Anão \\
\hline CNPGL 00-1-5 & Híbrido Intraespecífico & Tetraplóide & Anão \\
\hline CNPGL 00-16-1 & Híbrido Interespecífico & Triplóide $^{1}$ & Normal \\
\hline CNPGL $00-17-1$ & Híbrido Interespecífico & Triplóide $^{1}$ & Normal \\
\hline CNPGL 00-201-1 & Híbrido Interespecífico & Triplóide $^{1}$ & Normal \\
\hline CNPGL 00-206 & Híbrido Interespecífico & Hexaplóide $^{2}$ & Normal \\
\hline CNPGL $00-210$ & Híbrido Interespecífico & Hexaplóide $^{2}$ & Normal \\
\hline CNPGL $00-211$ & Híbrido Interespecífico & Hexaplóide $^{2}$ & Normal \\
\hline CNPGL $00-213$ & Híbrido Interespecífico & Hexaplóide $^{2}$ & Normal \\
\hline CNPGL 00-214 & Híbrido Interespecífico & Hexaplóide $^{2}$ & Normal \\
\hline CNPGL 00-219 & Híbrido Interespecífico & Hexaplóide $^{2}$ & Normal \\
\hline CNPGL $00-220$ & Híbrido Interespecífico & Hexaplóide $^{2}$ & Normal \\
\hline CNPGL 00-33-1 & Híbrido Interespecífico & Triplóide $^{1}$ & Normal \\
\hline CNPGL 00-64-1 & Híbrido Interespecífico & Triplóide $^{1}$ & Normal \\
\hline CNPGL $00-78-1$ & Híbrido Interespecífico & Triplóide $^{1}$ & Normal \\
\hline CNPGL $00-90-1$ & Híbrido Interespecífico & Triplóide $^{1}$ & Normal \\
\hline CNPGL $00-90-2$ & Híbrido Interespecífico & Triplóide $^{1}$ & Normal \\
\hline CNPGL 00-90-3 & Híbrido Interespecífico & Triplóide $^{1}$ & Normal \\
\hline CNPGL 91-11-2 & Híbrido Intraespecífico & Tetraplóide & Normal \\
\hline CNPGL 91-28-1 & Híbrido Intraespecífico & Tetraplóide & Normal \\
\hline CNPGL 93-25-3 & Híbrido Intraespecífico & Tetraplóide & Normal \\
\hline CNPGL 96-24-1 & Híbrido Intraespecífico & Tetraplóide & Normal \\
\hline Cameroom & Progenitor & Testemunha & Normal \\
\hline
\end{tabular}

O número de folhas por perfilho (NFP) foi determinado pela contagem das folhas de cinco perfilhos escolhidos aleatoriamente, desde a primeira folha viva até a última folha expandida. O número de perfilhos basais foi obtido através da contagem de todos os perfilhos existentes na parcela, dividido pela área da parcela $\mathrm{em} \mathrm{m}^{2}\left(\mathrm{NP}\right.$, perfilho $\mathrm{m}^{-2}$ ). $\mathrm{O}$ diâmetro do colmo (DC, $\mathrm{mm}$ ) foi medido com o auxílio de um paquímetro na base de cinco plantas por parcela. A altura da planta (AP, m) foi mensurada com auxílio de uma régua graduada, medindo do nível do solo até o ponto de curvatura da última folha expandida.

O delineamento experimental foi feito em blocos ao acaso, dado a declividade da área. Os dados foram submetidos à análise de variância e, caso fossem significativos, pelo teste de Fischer. A comparação entre médias foi realizada pelo teste de Scott-Knott (análise de agrupamento), e a relação entre as variáveis foi avaliada pela correlação de Pearson, ambas a 5\% de probabilidade.

Utilizou-se o seguinte modelo estatístico: $Y_{\mathrm{ijk}}: \mu+\mathrm{G}_{\mathrm{i}}+\mathrm{B}_{\mathrm{j}}+\alpha_{\mathrm{ijk}}$, sendo $\mu$ o efeito médio geral; $\mathrm{G}_{\mathrm{i}}$ o efeito do genótipo $\mathrm{i}, \mathrm{i}=25$ clones; $\mathrm{B}_{\mathrm{j}}$ o efeito do bloco $\mathrm{j}, \mathrm{j}=1$ e $2 ; \alpha_{\mathrm{ijk}}$ o erro aleatório associado ao genótipo i, bloco j no corte k. Utilizou-se o programa estatístico SISVAR $5.6^{(8)}$. 


\section{Resultados e discussão}

A altura das plantas (AP) foi agrupada em cinco grupos ( $\mathrm{P}>0,05)$, o clone CNPGL 00-103-1 ficou sozinho no grupo de maior altura, mesmo no segundo grupo de maiores alturas todos os clones superam dois metros (Tabela 2). Para esses clones, podemos sugerir que um menor intervalo entre cortes pode contribuir para reduzir a quantidade de componentes estruturais, tornando-os mais nutritivos para os animais. Pois, quando manejados com cortes até 45 dias de idade, Bhering et al. ${ }^{(9)}$ não observaram diferenças acentuadas na altura, diâmetro do colmo, percentagem de folhas e número de folhas do capim-elefante. $\mathrm{O}$ diâmetro do colmo diferiu em quatro grupos $(\mathrm{P}<0,05)$ (Tabela 2), onde apenas três clones ficaram no grupo com os maiores valores (CNPGL 00-219, CNPGL 00-90-2 e CNPGL 00-1-1) 1,2 ou 1,5 m.

Tabela 2 - Altura da planta (AP, m), diâmetro do colmo (DC, $\mathrm{mm})$, número de perfilhos (NP, perfilhos $\mathrm{m}^{-2}$ ), número de folhas por perfilho (NFP) e teor de matéria seca (TMS, \%) em clones de capim-elefante

\begin{tabular}{cccccc}
\hline Clone & AP $(\mathrm{m})$ & DC $(\mathrm{mm})$ & NP & NFP & TMS $(\%)$ \\
\hline CNPGL 00-103-1 & $2,30 \mathrm{a}$ & $11,50 \mathrm{~d}$ & $31,02 \mathrm{~b}$ & $14,58 \mathrm{a}$ & $23,51 \mathrm{a}$ \\
CNPGL 00-108-1 & $2,05 \mathrm{~b}$ & $14,00 \mathrm{~b}$ & $30,17 \mathrm{~b}$ & $12,45 \mathrm{a}$ & $19,98 \mathrm{~b}$ \\
CNPGL 00-1-1 & $1,88 \mathrm{c}$ & $16,59 \mathrm{a}$ & $11,42 \mathrm{~d}$ & $12,08 \mathrm{a}$ & $18,91 \mathrm{c}$ \\
CNPGL 00-1-5 & $1,90 \mathrm{c}$ & $12,80 \mathrm{c}$ & $33,47 \mathrm{~b}$ & $10,43 \mathrm{~b}$ & $18,91 \mathrm{c}$ \\
CNPGL 00-16-1 & $1,49 \mathrm{e}$ & $11,59 \mathrm{~d}$ & $8,17 \mathrm{~d}$ & $12,70 \mathrm{a}$ & $23,30 \mathrm{a}$ \\
CNPGL 00-17-1 & $2,06 \mathrm{~b}$ & $14,30 \mathrm{~b}$ & $16,63 \mathrm{c}$ & $14,53 \mathrm{a}$ & $18,68 \mathrm{c}$ \\
CNPGL 00-201-1 & $1,31 \mathrm{e}$ & $12,70 \mathrm{c}$ & $17,69 \mathrm{c}$ & $6,75 \mathrm{c}$ & $16,25 \mathrm{c}$ \\
CNPGL 00-206 & $1,68 \mathrm{~d}$ & $14,91 \mathrm{~b}$ & $20,14 \mathrm{c}$ & $14,55 \mathrm{a}$ & $17,91 \mathrm{c}$ \\
CNPGL 00-210 & $1,54 \mathrm{e}$ & $14,39 \mathrm{~b}$ & $16,32 \mathrm{c}$ & $10,70 \mathrm{~b}$ & $16,71 \mathrm{c}$ \\
CNPGL 00-211 & $1,83 \mathrm{c}$ & $12,28 \mathrm{c}$ & $38,42 \mathrm{a}$ & $11,78 \mathrm{a}$ & $18,11 \mathrm{c}$ \\
CNPGL 00-213 & $2,06 \mathrm{~b}$ & $12,43 \mathrm{c}$ & $31,01 \mathrm{~b}$ & $12,53 \mathrm{a}$ & $20,44 \mathrm{~b}$ \\
CNPGL 00-214 & $2,02 \mathrm{~b}$ & $14,35 \mathrm{~b}$ & $39,20 \mathrm{a}$ & $10,85 \mathrm{~b}$ & $18,03 \mathrm{c}$ \\
CNPGL 00-219 & $1,48 \mathrm{e}$ & $18,09 \mathrm{a}$ & $22,07 \mathrm{c}$ & $12,71 \mathrm{a}$ & $16,20 \mathrm{c}$ \\
CNPGL 00-220 & $1,55 \mathrm{e}$ & $15,29 \mathrm{~b}$ & $30,68 \mathrm{~b}$ & $8,85 \mathrm{~b}$ & $17,14 \mathrm{c}$ \\
CNPGL 00-33-1 & $1,86 \mathrm{c}$ & $10,23 \mathrm{~d}$ & $40,53 \mathrm{a}$ & $14,60 \mathrm{a}$ & $20,25 \mathrm{~b}$ \\
CNPGL 00-64-1 & $1,94 \mathrm{c}$ & $12,91 \mathrm{c}$ & $11,78 \mathrm{~d}$ & $12,38 \mathrm{a}$ & $20,39 \mathrm{~b}$ \\
CNPGL 00-78-1 & $2,14 \mathrm{~b}$ & $14,25 \mathrm{~b}$ & $16,11 \mathrm{c}$ & $14,88 \mathrm{a}$ & $20,64 \mathrm{~b}$ \\
CNPGL 00-90-1 & $1,58 \mathrm{e}$ & $15,15 \mathrm{~b}$ & $10,57 \mathrm{~d}$ & $13,60 \mathrm{a}$ & $17,58 \mathrm{c}$ \\
CNPGL 00-90-2 & $1,53 \mathrm{e}$ & $17,74 \mathrm{a}$ & $12,02 \mathrm{~d}$ & $6,51 \mathrm{c}$ & $16,53 \mathrm{c}$ \\
CNPGL 00-90-3 & $1,72 \mathrm{~d}$ & $13,20 \mathrm{c}$ & $12,54 \mathrm{~d}$ & $11,93 \mathrm{a}$ & $22,26 \mathrm{a}$ \\
CNPGL 91-11-2 & $1,67 \mathrm{~d}$ & $13,65 \mathrm{c}$ & $19,42 \mathrm{c}$ & $11,10 \mathrm{~b}$ & $20,09 \mathrm{~b}$ \\
CNPGL 91-28-1 & $1,65 \mathrm{~d}$ & $14,48 \mathrm{~b}$ & $33,46 \mathrm{~b}$ & $9,75 \mathrm{~b}$ & $17,88 \mathrm{c}$ \\
CNPGL 93-25-3 & $2,04 \mathrm{~b}$ & $11,93 \mathrm{~d}$ & $29,78 \mathrm{~b}$ & $15,00 \mathrm{a}$ & $21,79 \mathrm{a}$ \\
CNPGL 96-24-1 & $1,79 \mathrm{c}$ & $15,13 \mathrm{~b}$ & $26,87 \mathrm{~b}$ & $13,50 \mathrm{a}$ & $16,28 \mathrm{c}$ \\
Cameroom & $1,80 \mathrm{c}$ & $14,10 \mathrm{~b}$ & $25,14 \mathrm{~b}$ & $13,35 \mathrm{a}$ & $17,56 \mathrm{c}$ \\
\hline CV (\%) & 10,78 & 12,85 & 24,39 & 22,99 & 12,35 \\
\hline
\end{tabular}

Médias seguidas de letras distintas na coluna diferem entre si $(\mathrm{P} \approx 0,05)$ pelo teste de Scott-Knott. 
É comum os produtores colocarem os animais para pastejar capim-elefante com alturas maiores que $1,2 \mathrm{~m}$, porém esse manejo é prejudicial à planta. Segundo Schmitt et al. ${ }^{(10)}$, quando o capim-elefante é manejado com menos que $90 \mathrm{~cm}$, há vantagens na produção de forragem e no controle da estrutura do dossel, pois os bovinos não conseguem rebaixar mais do que metade dessa altura. Já segundo Viana et al. ${ }^{(11)}$, a altura ideal do capim-elefante para o pastejo de ovinos é de 0,72 m, obtidos nos clones Mott e Taiwan A-164 2.114, quando manejados com 32 dias de rebrotação. As alturas observadas mostram que nenhum dos clones avaliados são adequados ao pastejo.

$\mathrm{O}$ número de perfilhos $(\mathrm{NP})$ dividiu os clones em quatro grupos $(\mathrm{P}<0,05)$, grupo A com 3 clones com média de 39,38 perfilhos $\mathrm{m}^{-2}$; $\mathrm{B}$ com 9 clones e média de 30,18 perfilhos $\mathrm{m}^{-2}$; $\mathrm{C}$ com 7 clones e média de 18,34 perfilhos $\mathrm{m}^{-2}$ e D com 6 clones e média de 11,08 perfilhos $\mathrm{m}^{-2}$ (Tabela 2). Os grupos com as maiores quantidades de perfilhos sugerem a esses clones uma maior capacidade de rebrotação, além de maior cobertura do solo, contribuindo para uma menor invasão por plantas indesejáveis, que reduz os tratos culturais. O NP pode também ser um indicativo da produtividade do pasto, pois, segundo Hodgson $^{(12)}$, o perfilhamento é utilizado pela planta como forma de crescimento, aumento de produtividade e sobrevivência.

Em função do número de folhas por perfilho (NFP) os clones foram distribuídos em três grupos $(\mathrm{P}<0,05)$, em que 17 clones ficaram no grupo com maior quantidade (13,36 folhas), seguido do grupo intermediário com 6 clones (10,28 folhas) e no grupo com a menor quantidade foram alocados apenas os clones CNPGL 00-201-1 e CNPGL 00-90-2. (6,63 folhas por perfilho). O número máximo de folhas vivas por perfilho é uma constante genotípica pouco afetada por fatores abióticos ${ }^{(13)}$, quando o perfilho atinge seu número máximo de folhas vivas passa a haver um equilíbrio entre a taxa de surgimento e a taxa de senescência das folhas ${ }^{(14)}$, dessa maneira pode se definir essa variável como critério para determinar o momento de corte da planta e evitar perda da forragem produzida por senescência ${ }^{(15)}$.

A seleção de clones de Pennisetum sp. pelo teor de MS pode ser muito útil para o processo de ensilagem $^{(16)}$. Em estudo realizado por Monteiro et al. ${ }^{(17)}$, foi necessário utilizar $15 \%$ de farelo de arroz na massa de forragem ensilada para modificar o teor de matéria seca de 17,9 para 33,5\%, quanto maior o percentual de aditivos maior será o custo da ensilagem.

Os clones foram separados em três grupos em função dos teores de matéria seca $(\mathrm{P}<0,05)$, nos clones CNPGL 00-103-1, CNPGL 93-25-3, CNPGL 00-16-1 e CNPGL 00-90-3 foram observados os maiores valores, com média de $22,71 \%$ de MS. Os resultados mostram que esses clones podem ser mais adequados ao processo de ensilagem, uma vez que têm teores de matéria seca mais próximos ao ideal para ensilagem (30 a 35\% de MS). Nos demais clones, para esse fim, seria necessário maior tempo de desidratação, que poderia comprometer a qualidade da ensilagem ou mesmo, caso não sejam desidratados adequadamente, aumentar a quantidade de efluentes da silagem.

A massa de forragem (MFO) agrupou os clones em cinco grupos $(\mathrm{P}<0,05)$, no de maior produção ficou apenas com o clone CNPGL 00-214, enquanto que no grupo de menores MFO foram ficaram os clones CNPGL 00-16-1, CNPGL 00-90-1 e CNPGL 00-201-1, com média de 2373,3 kg ha-1 de MS a cada corte. Lima et al. ${ }^{(18)}$ encontraram produção de $14500 \mathrm{~kg} \mathrm{ha}^{-1}$ de MS para o cultivar Cameroon cortado a $30 \mathrm{~cm}$ do solo com 56 dias de rebrota no período chuvoso, essa produção foi cerca de $76 \%$ superior à obtida para a mesma cultivar neste trabalho. Essa diferença pode ser explicada pelo fato dos autores terem realizado o estudo com apenas um corte do capim e na estação 
chuvosa.

Tabela 3 - Valores médios para massa de forragem (MFO, $\mathrm{kg} \mathrm{ha}^{-1}$ de MS), de lâmina foliar (MLF, $\mathrm{kg} \mathrm{ha}^{-1}$ de MS), de colmo ((MCO, $\mathrm{kg} \mathrm{ha}^{-1}$ de MS) e relação lâmina foliar/colmo em clones de capimelefante

\begin{tabular}{ccccc}
\hline Clone & MFO & MLF & MCO & RLF/C \\
\hline CNPGL 00-103-1 & $9782,50 \mathrm{c}$ & $3758,75 \mathrm{c}$ & $6025,00 \mathrm{~b}$ & $0,66 \mathrm{~d}$ \\
CNPGL 00-108-1 & $11471,25 \mathrm{~b}$ & $4381,25 \mathrm{c}$ & $7092,50 \mathrm{~b}$ & $0,64 \mathrm{~d}$ \\
CNPGL 00-1-1 & $4580,00 \mathrm{e}$ & $1935,00 \mathrm{e}$ & $2645,00 \mathrm{~d}$ & $0,77 \mathrm{c}$ \\
CNPGL 00-1-5 & $10970,00 \mathrm{~b}$ & $4788,75 \mathrm{~b}$ & $6181,25 \mathrm{~b}$ & $0,81 \mathrm{c}$ \\
CNPGL 00-16-1 & $1835,00 \mathrm{f}$ & $796,25 \mathrm{e}$ & $1037,50 \mathrm{e}$ & $0,84 \mathrm{c}$ \\
CNPGL 00-17-1 & $5231,25 \mathrm{~d}$ & $2308,75 \mathrm{~d}$ & $2923,75 \mathrm{~d}$ & $0,80 \mathrm{c}$ \\
CNPGL 00-201-1 & $2451,25 \mathrm{f}$ & $1258,75 \mathrm{e}$ & $1192,50 \mathrm{e}$ & $1,17 \mathrm{a}$ \\
CNPGL 00-206 & $6520,00 \mathrm{~d}$ & $2545,00 \mathrm{~d}$ & $3972,50 \mathrm{c}$ & $0,66 \mathrm{~d}$ \\
CNPGL 00-210 & $4598,75 \mathrm{e}$ & $2052,50 \mathrm{e}$ & $2546,25 \mathrm{~d}$ & $0,83 \mathrm{c}$ \\
CNPGL 00-211 & $11567,50 \mathrm{~b}$ & $5095,00 \mathrm{~b}$ & $6472,50 \mathrm{~b}$ & $0,79 \mathrm{c}$ \\
CNPGL 00-213 & $9625,00 \mathrm{c}$ & $4151,25 \mathrm{c}$ & $5472,50 \mathrm{~b}$ & $0,76 \mathrm{c}$ \\
CNPGL 00-214 & $15852,50 \mathrm{a}$ & $6195,00 \mathrm{a}$ & $9657,50 \mathrm{a}$ & $0,65 \mathrm{~d}$ \\
CNPGL 00-219 & $6708,57 \mathrm{~d}$ & $3074,29 \mathrm{~d}$ & $3634,29 \mathrm{c}$ & $0,90 \mathrm{~b}$ \\
CNPGL 00-220 & $8121,25 \mathrm{c}$ & $3971,25 \mathrm{c}$ & $4150,00 \mathrm{c}$ & $0,99 \mathrm{~b}$ \\
CNPGL 00-33-1 & $7072,50 \mathrm{~d}$ & $3276,25 \mathrm{c}$ & $3803,75 \mathrm{c}$ & $0,86 \mathrm{c}$ \\
CNPGL 00-64-1 & $4077,50 \mathrm{e}$ & $1723,75 \mathrm{e}$ & $2352,50 \mathrm{~d}$ & $0,77 \mathrm{c}$ \\
CNPGL 00-78-1 & $7641,25 \mathrm{c}$ & $3365,00 \mathrm{c}$ & $4277,50 \mathrm{c}$ & $0,80 \mathrm{c}$ \\
CNPGL 00-90-1 & $2833,75 \mathrm{f}$ & $1350,00 \mathrm{e}$ & $1483,75 \mathrm{e}$ & $0,90 \mathrm{~b}$ \\
CNPGL 00-90-2 & $3731,43 \mathrm{e}$ & $1867,14 \mathrm{e}$ & $1864,29 \mathrm{~d}$ & $0,99 \mathrm{~b}$ \\
CNPGL 00-90-3 & $4173,75 \mathrm{e}$ & $1741,25 \mathrm{e}$ & $2432,50 \mathrm{~d}$ & $0,73 \mathrm{c}$ \\
CNPGL 91-11-2 & $5671,25 \mathrm{~d}$ & $2570,00 \mathrm{~d}$ & $3101,25 \mathrm{c}$ & $0,96 \mathrm{~b}$ \\
CNPGL 91-28-1 & $9013,75 \mathrm{c}$ & $4316,25 \mathrm{c}$ & $4697,50 \mathrm{c}$ & $0,95 \mathrm{~b}$ \\
CNPGL 93-25-3 & $9058,75 \mathrm{c}$ & $3061,25 \mathrm{~d}$ & $5997,50 \mathrm{~b}$ & $0,54 \mathrm{~d}$ \\
CNPGL 96-24-1 & $6853,75 \mathrm{~d}$ & $3015,00 \mathrm{~d}$ & $3838,75 \mathrm{c}$ & $0,80 \mathrm{c}$ \\
Cameroom & $8241,25 \mathrm{c}$ & $3761,25 \mathrm{c}$ & $4481,25 \mathrm{c}$ & $0,85 \mathrm{c}$ \\
\hline CV (\%) & 30,49 & 31,96 & 31,43 & 14,62 \\
\hline
\end{tabular}

Médias seguidas de letras distintas na coluna diferem entre si $(\mathrm{P}<0,05)$ pelo teste de Scott-Knott.

Gimenes et al. ${ }^{(19)}$ observaram produção de 2541 e $3623 \mathrm{~kg} \mathrm{ha}^{-1}$ de MS, para os clones CNPGL 9624-1 e CNPGL 91-11-2 respectivamente. Os resultados obtidos no presente trabalho foram quase duas vezes maiores que o relatado pelos autores, essa superioridade pode ser atribuída à utilização de irrigação e a temperatura mínima superior aos $20{ }^{\circ} \mathrm{C}$ durante todo experimento. Pois, segundo Villa Nova et al. ${ }^{(20)}$, as gramíneas tropicais necessitam de temperaturas dentro de faixas adequadas para alcançarem altas produções, sendo mínima de 12 a $15^{\circ} \mathrm{C}$, valores obtidos por Gimenes et al. ${ }^{(19)}$. Os valores de MFO mostram uma grande variação entre clones, este aspecto mostra sua importância no estudo de genótipos de capim-elefante sob mesma condição de adubação e irrigação, pois, os clones mais produtivos são mais eficientes economicamente em produção de forragem para alimentação animal.

Assim como na MFO, as maiores $(\mathrm{P}<0,05)$ massas de lâmina foliar (MLF) e de colmo (MCO) foram observadas no clone CNPGL 00-214. Segundo Martuscello et al. ${ }^{(21)}$, a alta e significativa correlação entre os componentes morfológicos do capim-elefante pode ser um indicativo de que o acúmulo de 
lâminas tende a aumentar a taxa fotossintética.

A relação lâmina foliar/colmo $(\mathrm{RLF} / \mathrm{C})$ agrupou os clones em quatro grupos $(\mathrm{P}<0,05)$ (Tabela 3$)$, em que no clone CNPGL 00-201-1 foi observado o maior valor, e apenas neste a massa de lâmina foliar foi superior à de colmo. Esse resultado corrobora os de Lima et al. ${ }^{(18)}$, em que os clones com maiores $\mathrm{RLF} / \mathrm{C}$ foram os menos produtivos. Os autores avaliaram 10 genótipos de capim-elefante e destacaram que o fato de alguns genótipos não alcançarem melhores relações RLF/C não compromete a seleção, uma vez que a tendência é que a adaptação dos híbridos melhore com o passar do tempo.

A RLF/C é fator de predição da qualidade da forragem, pois o valor nutritivo do caule é inferior ao da folha, logo, maior massa de folhas em relação à de colmos é uma característica desejável à forrageira por estar diretamente relacionada à qualidade e ao consumo, fato ainda mais relevante em forragens com colmos mais espessos, como o capim-elefante. Vale ressaltar que os resultados observados podem ser diferentes para os mesmos híbridos em outros climas e com diferentes níveis e/ou fontes de adubação.

Embora muitos dos coeficientes das correlações avaliadas tenham sido significativos $(\mathrm{P}<0,05)$, poucos foram de média ou alta magnitude (Tabela 4). Isso pode ser atribuído à diversidade genética entre os clones, caso esta análise fosse realizada apenas com um clone, poderíamos obter maiores coeficientes de correlação. A MFO foi a variável com os maiores coeficientes de correlação com as demais, destacando-se sua alta e positiva correlação com o NP, a MLF e a MCO. Essas associações já eram esperadas, uma vez que o NP é a forma de crescimento da planta e que as MLF e MCO são derivadas da MFO.

Tabela 4 - Coeficientes de correlação de Pearson entre variáveis morfológicas e produtivas em clones de capim-elefante

\begin{tabular}{cccccccccc}
\hline & NFP & AP & DC & TMS & MFO & NP & RLF/C & MLF & MCO \\
\hline NFP & - & $0,24^{*}$ & $-0,27^{*}$ & $0,41^{*}$ & $-0,06^{\text {ns }}$ & $-0,02^{\text {ns }}$ & $-0,24^{*}$ & $-0,10^{\text {ns }}$ & $-0,04^{\text {ns }}$ \\
AP & - & - & $0,04^{\text {ns }}$ & $0,00^{\text {ns }}$ & $0,55^{*}$ & $0,34^{*}$ & $-0,49^{*}$ & $0,47^{*}$ & $0,58^{*}$ \\
DC & - & - & - & $-0,42^{*}$ & $0,12^{\text {ns }}$ & $-0,17^{*}$ & $0,04^{\text {ns }}$ & $0,14^{*}$ & $0,10^{\text {ns }}$ \\
TMS & - & - & - & - & $-0,10^{\text {ns }}$ & $-0,19^{*}$ & $-0,14^{\text {ns }}$ & $-0,14^{\text {ns }}$ & $-0,07^{\text {ns }}$ \\
MFO & - & - & - & - & - & $0,73^{*}$ & $-0,40^{*}$ & $0,97^{*}$ & $0,99^{*}$ \\
NP & - & - & - & - & - & - & $-0,22^{*}$ & $0,72^{*}$ & $0,70^{*}$ \\
RLF/C & - & - & - & - & - & - & - & $-0,22^{*}$ & $-0,50^{*}$ \\
MLF & - & - & - & - & - & - & - & - & $0,92^{*}$ \\
MCO & - & - & - & - & - & - & - & - & - \\
\hline
\end{tabular}

${ }^{\text {× }}$, não significativo; *, $\mathrm{P}=0,05$. NFP, número de folhas por perfilho; $\mathrm{AP}$, altura da planta; $\mathrm{DC}$, diâmetro do colmo; TMS, teor de matéria seca; MFO, massa de forragem; NP, número de perfilhos; RLF/C, relação lâmina foliar/colmo; $M L F$, massa de lâmina foliar; MCO, massa de colmo.

A AP correlacionou-se positivamente com a MFO, MLF e MCO. Assim, com o aumento da AP ocorre também aumento dessas variáveis, porém, podemos observar que a correlação entre a AP e a MCO é superior à da MLF, confirmado pela correlação negativa da AP com a RLF/C. A correlação positiva entre AP e MFO também foram observadas por Cunha et al. ${ }^{(16)}$, o que ratifica a associação mesmo em diferentes ambientes. Essa associação acontece porque o colmo é o componente estrutural responsável pela sustentação e pelo aumento da altura, em função do alongamento do mesmo. A facilidade na medição de altura das plantas e sua correlação com parâmetros de produção fazem com 
que esta seja uma ferramenta potencial para o manejo de gramíneas tropicais ${ }^{(22)}$. Segundo Emerenciano Neto et al. ${ }^{(23)}$, o aumento da altura da planta promove aumento na massa de forragem, porém, aumenta significativamente a participação de colmo e material morto nessa massa.

\section{Conclusões}

O clone CNPGL 00-214 mostrou-se mais produtivo, com alta capacidade de perfilhamento e de acúmulo de forragem, porém, a baixa relação lâmina foliar/colmo deste clone sugere que sejam avaliados manejos com menores intervalos de corte para melhoria qualitativa da forragem produzida.

\section{Agradecimentos}

A Universidade Federal do Rio Grande do Norte - UFRN, pela execução do experimento, Empresa de Pesquisa Agropecuária do Rio Grande do Norte - EMPARN, pelo local cedido e condução do experimento e a Empresa Brasileira de Pesquisa Agropecuária - EMBRAPA, através do Programa RENACE (Rede Nacional de Avaliação do Capim-elefante) pelos materiais de estudo.

\section{Referências}

1. Cunha MV, Lira MA, Santos MVF, Dubeux Júnior JCB, Mello ACL, Freitas EV. Adaptabilidade e estabilidade da produção de forragem por meio de diferentes metodologias na seleção de clones de Pennisetum spp. Revista Brasileira de Ciências Agrárias. 2013, 8(4): 681-686. http://dx.doi.org/10.5039/agraria.v8i4a3280

2. Leão FF, Cancellier LL, Pereira AV, Ledo FJS, Afférri FS. Produção forrageira e composição bromatológica de combinações genômicas de capim-elefante e milheto. Revista Ciência Agronômica. 2012, 43(2):368-375. http://dx.doi.org/10.1590/S180666902012000200021

3. Silva MC, Santos MVF, Lira MA, Mello ACL, Freitas EV, Santos RJM, Ferreira RLC. Ensaios preliminares sobre autofecundação e cruzamentos no melhoramento do capim-elefante. Revista Brasileira de Zootecnia. 2008, 37(3):401-410. http://dx.doi.org/10.1590/S1516-35982008000300004

4. Santos AE, Silva DS, Queiroz Filho JL. Aspectos produtivos do capim-elefante (Pennisetumpurpureum, Schum.) cv. Roxo no brejo paraibano. Revista Brasileira de Zootecnia. 2001, 30(1): 31-36. http://dx.doi.org/10.1590/S1516-35982001000100006

5. Silva ALC, Santos MVF, Dubeux Junior, J.C.B. Lira, M.A., Ferreira, R.L.C., Freitas, E.V., Cunha, M.V., Silva, M.C. Variabilidade e herdabilidade de caracteres morfológicos em clones de capim-elefante na Zona da Mata de Pernambuco. Revista Brasileira de Zootecnia, v.39, n.10, p.2132-2140, 2010. http://dx.doi.org/10.1590/S1516-35982010001000005.

6. Empresa Brasileira de Pesquisa Agropecuária (EMBRAPA). (2006). Sistema brasileiro de classificação de solos (2nd ed., p. 306). Rio de Janeiro.

7. Thornthwaite CW. An approach toward a rational classification of climate. Geographical Review. 1948, 38:55-94. https://doi.org/10.2307/210739

8. Ferreira DF. Sisvar: a computer statistical analysis system. Ciência e Agrotecnologia, 2011, 35(6):10391042. https://doi.org/10.1590/S1413-70542011000600001 
9. Bhering M, Cabral LS, Abreu JG, Souza AL, Zervoudakis JT, Rodrigues RC, Pereira GAC,Reverdito R, Oliveira IS. Características agronômicas do capim-elefante roxo em diferentes idades de corte na Depressão Cuiabana. Revista Brasileira de Saúde e Produção Animal. 2008, 9(3):384-396. http://revistas.ufba.br/index.php/rbspa/article/viewArticle/1234

10. Schmitt D, Padilha DA, Dias KM, Santos GT, Rodolfo GR, Zanini GD, Sbrissia AF. Chemical composition of two warm-season perennial grasses subjected to proportions of defoliation. Grassland Science. 2019, 0:1-8. https://doi.org/10.1111/grs.122366

11. Viana BL, Mello AC Leão, Guim A, Lira MA, Dubeux Júnior JCB, Santos MVF, Cunha MV. Morphological characteristics and proportion of leaf blade tissues of elephant grass clones under sheep grazing. PesquisaAgropecuária Brasileira. 2018, 53(11):1268-1275. http://dx.doi.org/10.1590/s0100$\underline{204 \times 2018001100009}$

12. Hodgson J. Grazing Management: Science into practice. New York: John Wiley \& Sons. p. 203, 1990.

13. Edvan RL, Santos EMA, Silva DSB, Andrade AP, Costa RG, Vasconcelos WA. Características de produção do capim-buffel submetido a intensidades e frequências de corte. Archivos de Zootecnia.2011, 60(232):1281-1289. http://dx.doi.org/10.4321/S0004-05922011000400043

14. Fulkerson WJ, Slack K, Havilah E. The effect of defoliation interval and height on growth and herbage quality of kikuyu grass (Pennisetumclandestinum). Tropical Grassland, 1999, 33(3):138-145.

15. Luna AA, Difante GS, Montagner DB, Emerenciano Neto JV, Araújo IMM, Oliveira LEC. Características morfogênicas e acúmulo de forragem de gramíneas forrageiras sob corte. BioscienseJournal.2014, 30(6):18031810 .

16. Cunha MV, Lira MA, Santos MVF, Freitas EV, Dubeux Junior JCB, Mello ACL, Martins KGR. Association between the morphological and productive characteristics in the selection of elephant grass clones. Revista Brasileira de Zootecnia. 2011, 40(3):482-488. http://dx.doi.org/10.1590/S1516-35982011000300004

17. Monteiro IJG, Abreu JG, Cabral LS, Almeida RG, Reis RHP, Behling Neto A, Cabral CEA, Barros LV, Avelino ACD,Penso S. Ensiling of elephant grass with soybean hulls or rice bran. Semina: Ciências Agrárias.2016, 37(6):4203-4212. http://dx.doi.org/10.5433/1679-0359.2016v37n6p4203

18. Lima ES, Silva JFC, Vásquez HM, Araújo SAC, Lista FN, Costa DPB. Produção de matéria seca e proteína bruta e relação folha/colmo de genótipos de capim-elefante aos 56 dias de rebrota. Revista Brasileira de Zootecnia.2007, 36(5):1518-1523. http://dx.doi.org/10.1590/S1516-35982007000700009

19. Gimenes FMA, Berndt A, Mateus GP, Ledo FJS,Demarchi JJAA, Giacomini AA, Gerdes L, Batista K. Forage mass and agronomic traits of Elephant grass genotypes under free-growth and conventional cutting systems. Semina: Ciências Agrárias. 2016, 37(4):2577-2588. http://dx.doi.org/10.5433/16790359.2016v37n4Sup11p2577

20. Villa Nova NA, Tonato F, Pedreira CGS, Medeiros HR. Método alternativo para cálculo da temperatura base de gramíneas forrageiras. Ciência Rural. 2007, 37(2):545-549. http://dx.doi.org/10.1590/S0103$\underline{84782007000200039}$

21. Martuscello JA, Majerowicz N, Cunha DNFV, Amorim PL, Braz TGS. Características produtivas e fisiológicas de capim-elefante submetido à adubação nitrogenada. Archivos de Zootecnia. 2016, 65(252):565570.

22. Difante GS, Euclides VPB, Nascimento Júnior D, Silva SC, Barbosa RA, Torres Júnior RAA. Desempenho e conversão alimentar de novilhos de corte em capim-tanzânia submetido a duas intensidades de pastejo sob lotação rotativa. Revista Brasileira de Zootecnia. 2010, 39:33-41. http://dx.doi.org/10.1590/S1516$\underline{35982010000100005}$ 
23. Emerenciano Neto JV, Difante GS, Lana AMQ, Campos NRF, Veras ELL, Moraes JD. Sward structure and herbage accumulation of massaiguineagrass pastures managed according to pre-grazing heights, in the northeast of Brazil. Journal of Agricultural Science, 2017, 9:155-163. http://dx.doi.org/10.5539/jas.v9n4p155 